\title{
Une expérience transculturelle. Entretien avec Gerhard Kubik
}

\section{Tiago de Oliveira Pinto et Gerhard Kubik}

Traducteur : Isabelle Schulte-Tenckhoff

\section{(2) OpenEdition \\ Journals}

Édition électronique

URL : http://journals.openedition.org/ethnomusicologie/1411

ISSN : 2235-7688

Éditeur

ADEM - Ateliers d'ethnomusicologie

Édition imprimée

Date de publication : 31 décembre 1994

Pagination : 211-227

ISBN : 2-8257-0503-9

ISSN : $1662-372 X$

Référence électronique

Tiago de Oliveira Pinto et Gerhard Kubik, « Une expérience transculturelle. Entretien avec Gerhard Kubik », Cahiers d'ethnomusicologie [En ligne], 7| 1994, mis en ligne le 03 janvier 2012, consulté le 03 mai 2019. URL : http://journals.openedition.org/ethnomusicologie/1411

Ce document a été généré automatiquement le 3 mai 2019.

Tous droits réservés 


\title{
Une expérience transculturelle. Entretien avec Gerhard Kubik*
}

\author{
Tiago de Oliveira Pinto et Gerhard Kubik \\ Traduction : Isabelle Schulte-Tenckhoff
}

1 En 1975 - j'étais encore écolier - un ami me parla d'une conférence que Gerhard Kubik avait prononcée au Goethe-Institut de ma ville natale, São Paulo, et d'un concert qu'il avait donné avec deux musiciens du Malawi dans l'auditoire du Museu de Arte de la même ville. A l'époque, $\mathrm{j}$ 'avais de la peine à m'imaginer un chercheur européen qui, après avoir vécu et travaillé dans différents pays africains, était venu au Brésil pour retracer les liens historiques avec l'Afrique, notamment dans le domaine musical et culturel - mais je n'en étais pas moins fasciné.

2 J'ai finalement rencontré Kubik pour la première fois en 1984 pendant mes études à l'Université Libre de Berlin. Depuis, nous nous sommes revus chaque année pour échanger nos idées. L'amitié que j'ai pu développer au cours des dix dernières années avec Lidiya, la regrettée épouse de Kubik, ainsi qu'avec ses collègues Moya Aliya Malamusi, Donald Kachamba et Mose Yotamu représente pour moi une expérience particulièrement instructive.

3 Le présent entretien est un montage établi à partir d'extraits de plusieurs longues conversations menées durant l'été 1993 à Berlin. Cette publication, qui marque les soixante ans de Gerhard Kubik, est destinée à rendre hommage à sa personnalité exceptionnelle et fascinante et à intéresser les jeunes chercheurs à son œuvre.

4 Ayant dû renoncer, par manque de place, à reproduire l'importante bibliographie de Kubik (quelque 300 titres), je renvoie le lecteur intéressée à la Festschrift qui lui est dédiée (Födermayr et al. 1994), ainsi qu'au recueil le plus récent de notre auteur (Kubik 1994).

Gerhard Kubik est né le 10 décembre 1934 à Vienne. Adolescent, il commence à pratiquer la musique, en s'intéressant tout particulièrement au swing, puis au bebop - expérience qui s'avérera déterminante pour son premier voyage en Afrique. Dès 1954, Kubik se produit en public avec son ensemble de jazz « Musici »; en 1959, il obtient le premier prix du Festival de jazz de Vienne. Kubik est étudiant (africanisme et science politique) 
lorsque son ensemble de jazz se dissout en 1959. Cet incident pousse Kubik à entreprendre son premier voyage en Afrique, en octobre 1959. Ne disposant que de quatre-vingts livres sterling, il se rend en auto-stop de Vienne en Afrique orientale, en traversant l'ex-Yougoslavie, la Grèce, l'Égypte et le Soudan. Dans ses bagages, il y a déjà un enregistreur. Ce premier voyage dure une année : Gerhard Kubik entreprend alors ses premières recherches de terrain sur la musique en Ouganda, au Tanganyika, au Congo, au Cameroun et au Nigéria.

T.de O.P.

La longue durée de votre premier voyage en Afrique était-elle prévue?

Autant que je me souvienne, je n'avais pas de projet précis, seulement une très forte motivation. A ce stade, le voyage en Afrique était ma seule chance de survie psychique. Mon groupe de jazz s'étant dissout, je cherchais à m'intégrer à une autre communauté musicale. A l'origine, j'avais l'intention de me rendre en Afrique de l'Ouest, car pendant mes années d'activité en tant que musicien de jazz, je m'étais familiarisé avec les disques de musique ouest-africaine disponibles à l'époque, ainsi qu'avec la littérature s'y rapportant. J'étais devenu membre de l'African Music Society, et je m'étais adressé à Hugh Tracey pour l'informer de mon projet de recherche. Tracey a été le seul qui m'ait encouragé à l'entreprendre, alors que mes professeurs de l'Université de Vienne, sans me décourager explicitement, s'étaient montrés sceptiques et inquiets. D'après eux, je devais terminer mes études avant de m'engager dans une recherche de terrain. Influencé par les travaux de Richard A. Waterman et les disques enregistrés par Gilbert Rouget en Afrique occidentale, surtout en Guinée, ainsi que par le courant qui, à l'époque, cherchait des « racines » ouest-africaines au jazz, j'avais décidé de me rendre en Afrique occidentale. Mais à cause de la guerre d'Algérie, ce n'était pas possible par voie terrestre. Comme je ne pouvais voyager que de cette façon-là, ayant peu de moyens financiers, j'ai choisi la route est-africaine. Le voyage de Vienne à Kampala a duré sept semaines.

Le hasard a voulu qu'en Ouganda, je rencontre rapidement un professeur prêt à me donner un premier enseignement pratique et régulier relatif à une culture musicale africaine. C'était Evaristo Muyinda, un musicien attaché à la cour du kabaka (régent) de Buganda.

Vous comptiez trouver une nouvelle communauté musicale. Qu'est-ce que cela signifiait pour vous? La pratique musicale était-elle prédominante, ou plutôt la volonté de vous intégrer à une communauté socioculturelle?

C'était les deux. Dans mon ensemble de jazz, le sentiment d'appartenance ne se limitait pas à la pratique collective de la musique. Ses membres, du moins certains d'entre eux, partageaient d'autres intérêts, comme celui pour l'art contemporain. L'un était peintre, il l'est d'ailleurs toujours. Un autre domaine d'intérêt commun était la littérature. J'écrivais moi-même des poèmes et de la prose [Kubik \& Andika 1967]. Cette expérience, archétype d'une sorte de communauté de classe d'âge, pouvait être projetée aisément sur l'Afrique ; et sans m'en rendre compte, j'ai cherché dès le début à être intégré à un groupe analogue de sympathisants et d'alliés. C'est pour cette raison que j'ai gardé mes distances, dans l'Afrique orientale et centrale de 1960, vis-à-vis des Européens des colonies qui cherchaient à se différencier de la population locale. J'ai résisté par tous les moyens possibles à leurs tentatives de m'intégrer à leur communauté1. 
Que signifiait pour vous l'enseignement d'Evaristo Muyinda, éminente personnalité musicale est-africaine?

Comme c'était à prévoir, les premiers mois en Ouganda et l'enseignement d'Evaristo Muyinda ont provoqué en moi un choc culturel considérable. Rétrospectivement, je me dis que je l'ai plutôt bien vécu. Mon profil culturel personnel au moment de la dissolution du groupe de jazz était celui d'un homme aux racines littéraires et scientifiques européennes, mais aux racines musicales plutôt afro-américaines... Rapidement en Ouganda, nombre de comportements et de conceptions que j'avais acquis comme musicien de jazz se sont avérés inutiles et non pertinentes. Evaristo s'est mis à m'enseigner le jeu du xylophone. Notre communication était fort laborieuse, car le luganda, la langue maternelle d'Evaristo Muyinda, ne s'apprenait pas en quelques semaines, et Muyinda ne parlait pas l'anglais. C'est donc le kiswahili, que j'avais commencé à apprendre dans le cadre de mes études africanistes à Vienne, qui nous a servi de moyen de communication. Nous nous parlions en kiswahili, mais Muyinda l'agrémentait d'une terminologie musicale en luganda.

Fig. 1 : De g. à dr. : Donald Kachamba, Gerhard Kubik et Moya Aliya.

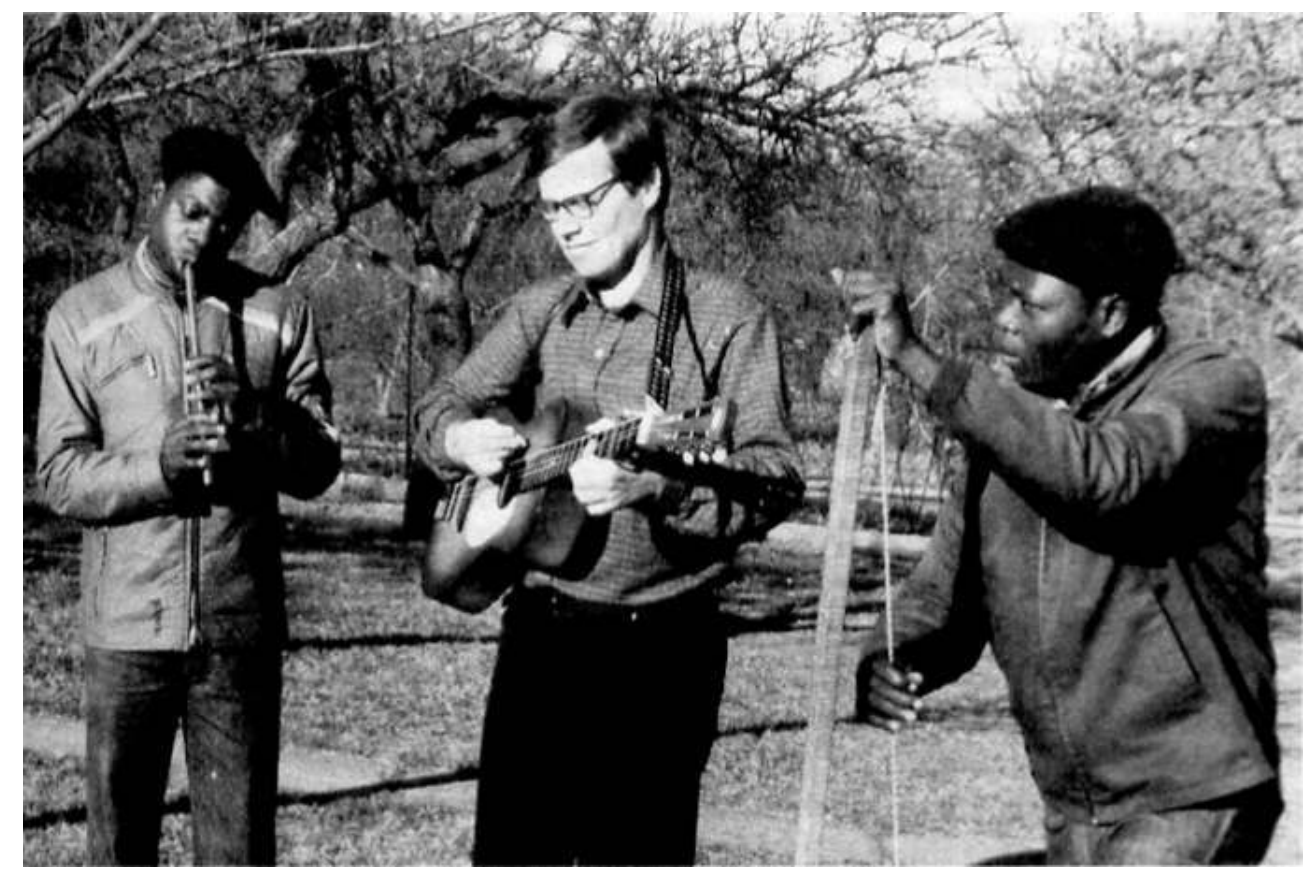

Photo : M. Hillegeist, 1987

Le premier choc est survenu lorsque Muyinda a commencé à m'enseigner le jeu du xylophone à douze lames, amadinda ; il m'a demandé de répéter sans cesse une série d'intervalles identiques, qu'il appelait okunaga. Je ne m'y attendais pas du tout. Je pensais trouver ce dont on parlait dans les livres: des formules et des rythmes complexes. Je me souviens encore qu'au début de cet enseigement je m'étais demandé si Muyinda me montrait « du vrai de vrai ». La partie okunaga me semblait trop simple, plus simple encore que les rythmes de la musique européenne. Cétait donc cela, l'Afrique ? Néanmoins, j'ai appris la série scrupuleusement et je l'ai frappée avec les deux maillets tenus parallèlement, selon des intervalles que Muyinda appelait miyanjo, mais que ma propre formation musicale m'amenait à considérer comme des « octaves ». Il restait à explorer si ces deux notions étaient conciliables. 
Quelle était votre relation personnelle avec Evaristo Muyinda?

Evaristo Muyinda était un enseignant d'une amabilité et d'une bonté extraordinaires. Très vite, il m'a imposé du respect, si bien que je me suis soumis entièrement à sa volonté. Il a commencé par tenir mes mains dans les siennes pour m'apprendre le bon toucher; il m'a indiqué que pour jouer ces miyanjo parallèles, je devais bouger davantage les poignets et moins les bras. Après quelques jours, il a semblé satisfait de la manière dont je jouais cette série de douze frappes. Il a donc pris ses propres maillets et, assis en face de moi de l'autre côté du xylophone à lames sur troncs de bananiers, il s'est mis à exécuter des frappes s'intercalant entre les miennes. Voilà qui me plaisait! J'avais le sentiment que ma partie seule, d'apparence si simple, ne comptait pas en tant que telle, que ce qui importait était une combinaison. En quoi consistait-elle? J'étais curieux. Muyinda a suggéré que nous changions de côté ; il voulait jouer ma partie, tandis que j'apprenais la sienne. Mais quand il me l'avait montrée, j'étais à nouveau déçu. Dans le jeu d'ensemble, le tout m'avait semblé fort complexe. Maintenant je m'apercevais qu'il ne s'agissait que de trois notes qu'il fallait répéter inlassablement. Retrospectivement, je vois que ma réaction initiale était celle d'un Occidental typique, impatient et avide d'improviser pour faire preuve de sa "créativité ». Voici donc la première leçon que j'ai reçue de Muyinda: se limiter au nécessaire, aspirer à l'économie, maîtriser son exubérance, observer un ordre et une discipline stricts. De toute évidence, il fallait que j'oublie cet Occidental qui somnolait encore en moi. C'est cela que visait l'enseignement. Ainsi, je me suis mis à apprendre la séquence de sons de la partie 2, que je devais jouer quatre fois pour remplir la durée de la partie 1 jouée maintenant par mon maître. Alors surgit un nouveau problème : Muyinda n'était pas satisfait de mon jeu, car nos frappes coïncidaient. Chaque fois, après m'avoir indiqué le début de ma partie, il essayait de retarder la sienne, mais je le poursuivais avec une insistance sur le synchronisme qui le surprenait. Enfin une lueur m'est venu : peut-être fallait-il que ma partie 2 s'insère dans la sienne, qu'elle syncope continuellement. Muyinda a joué la partie 1 très lentement pendant que j'y insérais la partie 2, tout en exécutant des syncopes. Une fois, je murmurais si fort le rythme imaginaire sur lequel je modelais mes syncopes que Muyinda et quelques élèves qui se trouvaient là en étaient très amusés. Nul doute, je ne jouais pas juste, car je perdais le temps dès que Muyinda accélérait sa partie. Pour finir, Muyinda a trouvé un remède. Il m'a demandé de commencer seul la partie 2 et de la jouer comme j'avais joué auparavant la partie 1 . J'exécutais donc les frappes de la partie 2 sans recourir à la syncope, simplement dans le rythme, pour ainsi dire « sur le temps ». Muyinda a intercalé les frappes de la partie 1 entre les miennes, et subitement ça marchait! Le résultat était magnifique et fort complexe. En l'écoutant, on pouvait s'y noyer comme dans un rêve. J'aurais pu continuer à jouer pendant des heures. Dans cet univers que nous partagions, lui et moi, il n'y avait plus qu'un lien relatif. Nous nous regardions comme dans une réalité "flottante ». C'est ainsi que j'ai découvert ce que Arthur M. Jones avait déjà décrit en 1934, en termes de cross rhythms, ce qu'on appelle aujourd'hui le beat de référence relative. J'ai découvert que dans cette musique, et contrairement au jazz, on ne partait pas d'un temps fort de référence, mais que chacun devait trouver sa position par rapport à son vis-à-vis. Il importait que les frappes de la partie 2 jouée par moi tombent, chaque fois en l'espace d'une fraction de seconde, entre les frappes de la partie 1 exécutée par mon vis-à-vis. Il fallait que je joue comme si je me trouvais au centre d'un champ de gravitation. Ainsi y a-t-il abandon de toute notion de syncope. 
.et vous avez accédé à un monde « autre ».

C'est exact en quelque sorte. En 1959, vers la mi-décembre, Muyinda, content des progrès que j'avais faits dans la compréhension de sa musique, s'est mis à m'enseigner le jeu du deuxième xylophone de Buganda, instrument plus grand appelé akadinda. J'ai compris que, dans ce cas également, le principe du beat relatif était valable et que je devais tomber avec mes frappes (dans ce cas, il s'agissait en fait de deux frappes) entre les siennes. Dans un centre agricole pour aveugles près de Kampala, où mon maitre enseignait régulièrement, j'ai fini par trouver le partenaire idéal pour pratiquer la musique de l'akadinda. La cécité de mon partenaire avait un effet bénéfique pour notre association, étant donné que les rapports humains restaient teintés de racisme (encore que la situation en Ouganda fût, de ce point de vue, meilleure qu'au Kenya de l'époque) : privés de contact visuel, nous étions égaux. Pendant cette phase finale de la domination britannique, j'ai trouvé dans cette niche culturelle formée par l'ensemble d'akadinda des aveugles une résurgence de la communauté musicale que j'avais perdue en été 1959 à Vienne.

Déjà en décembre 1959, l'enseignement musical de Muyinda devait me réserver un autre choc culturel. Muyinda m'a appris que toutes les pièces pour akadinda exigeaient une troisième partie qu'il fallait jouer sur les lames les plus aiguës du xylophone. A ma grande surprise, cette troisième partie était en fait une sorte de formule, comme je me l'étais imaginé à la lecture d'ouvrages sur la musique d'Afrique occidentale. Or, en particulier dans les pièces plus longues, ces formules étaient parfois si complexes qu'elles dépassaient mes capacités de mémorisation. Muyinda a commencé à m'apprendre systématiquement quelques-unes de ces formules accompagnant les douzaines de compositions pour amadinda que je connaissais déjà. Je les ai apprises aussi bien que possible, mais j'ignorais comment trouver une sorte d'orientation rythmique pour ces formules complexes. Quand je m'imaginais un beat sous-jacent, comme je l'avais fait dans mon ensemble de jazz, ces formules me semblaient être à contretemps (off-beat) d'une manière si complexe qu'il devenait quasi impossible de les mémoriser, étant donné que leur écart mélodique se limitait à deux tons et que l'emplacement des points d'impact était tout à fait irrégulier. Je l'ai bien compris lorsque Muyinda s'est mis à jouer avec un autre élève les deux parties de base, me demandant de jouer la formule - qu'il appelait okukoonera - sur les deux lames les plus aiguës du xylophone. Régulièrement je ratais la combinaison. Comme un film mal synchronisé, nos parties s'écartaient infailliblement. Je désespérais. Voyant que j'étais triste, Muyinda est venu encore une fois à mon secours. Il m'a expliqué que, pour entendre correctement l'okukoonera, il me fallait prêter attention aux sons émis par les deux lames les plus graves, qu'il appelait amatengezzi. Lorsque Muyinda et son élève ont recommencé à jouer, je n'ai tout d'abord rien entendu. Alors ils ont fait ressortir de l'ensemble sonore les notes produites par les deux lames graves. Tout d'un coup, j'ai eu un déclic : voilà l'okukoonera, voilà ce que j'étais censé jouer, seulement deux octaves plus bas. Lentement je comprenais, à propos de cette partie 3 qu'Evaristo Muyinda m'avait enseignée dans toute sa complexité rythmique, qu'il fallait simplement la faire coïncider avec les sons émis par les deux lames les plus graves.

J'ai vécu encore un autre choc, qui a provoqué une modification décisive de mon profil culturel personnel. Sur un enregistrement des parties 1 et 2 jouées ensemble, ma partie avait disparu. En écoutant la bande, voire la combinaison des deux parties, on ne distinguait plus les parties en tant que telles. Quelque chose de tout à fait nouveau était 
né, une structure qui oscillait, qui fluctuait, qui pouvait changer d'apparence comme une image holographique. C'était une structure dans laquelle je pouvais entendre au moins deux, parfois trois phrases mélodico-rythmiques entrelacées, qui s'imposaient à tel point à la conscience qu'il était impossible d'en faire abstraction et d'en ignorer la configuration. A ma plus grande surprise, une de ces phrases était exactement la formule sur laquelle mon maître avait attiré mon attention quand j'avais essayé en vain de jouer la partie 3. C'était la formule émise par les deux lames les plus graves du xylophone. Personne d'entre nous ne l'avait pourtant jouée. J'ai appelé cette découverte inherent rhythms ou subjective rhythms (rythmes inhérents ou subjectifs).

Ces expériences ont complètement réorienté votre vie : vous étiez sur le point d'embrasser une carrière scientifique. Déjà deux ans plus tard, le 28 mars 1963, vous avez prononcé au Royal Anthropological Institute à Londres une conférence introduite par Klaus Wachsmann, intitulée «Xylophone-playing in southern Uganda ». L'apprentissage auprès de M. Muyinda, qui n'était qu'un parmi les nombreux maîtres africains dont vous alliez suivre l'enseignement au fil des années, a eu des répercussions encore récemment: le compositeur Györgi Ligeti lui-même est venu vous trouver pour apprendre davantage sur les techniques de composition de l'amadinda ougandaise. D'autre part, vous venez de publier dans The World of Music un article sur la musique d'un autre xylophone ougandais, I' embaire de Busoga [Kubik 1992]. Or, quel que fut le succès des enseignements que vous avez suivis auprès de musiciens africains, où en étaient, dans les années soixante, vos études universitaires à Vienne? Et comment les spécialistes ont-ils réagi aux publications retentissantes d'un étudiant?

Pour ce qui est de mes études à Vienne, j'avais changé d'orientation en m'inscrivant en musicologie comparée et en études africanines, mais en 1963-64, après deux autres années de séjours ininterrompus sur le terrain en Afrique (27 août 1961 au 10 janvier 1963), je n'étais plus un étudiant que formellement, c'est-à-dire que je ne suivais plus de cours et ne passais plus d'examens. Très vite après mon retour en Europe, je me suis lancé dans les préparatifs d'un nouveau voyage d'exploration, entre autres en Afrique occidentale et centrale en 1963-64. Tout ce qui me liait encore à mon lieu de naissance était - à part ma mère - le souci d'incorporer régulièrement la totalité de mes enregistrements de terrain aux Archives sonores de l'Académie autrichienne des sciences à Vienne, dirigées à l'époque par Walter Graf.

Mon premier travail scientifique sur la musique africaine est paru en 1960 dans African Music sous le titre "The structure of Kiganda xylophone music ». Les réactions furent, soit très positives, ce qui fut le cas de A.M. Jones (dont j'ai d'ailleurs gardé une lettre bienveillante) et de Hugh Tracey, soit réservées comme chez Klaus Wachsmann, soit franchement négatives comme dans le cas de l'archéologue Merrick Posnansky².

Quelle a été votre propre formation universitaire? Qui étaient vos pères spirituels, si l'on peut dire? Ou plutôt, les études ne représentaient-elles pour vous qu'une formalité, vous considérez-vous comme une sorte d'autodidacte?

Sincèrement, dans cette première phase, ceux dont je m'inspirais étaient déjà décédés, et aucun d'entre eux n'appartenait à une institution universitaire. Je les connaissais plutôt par leurs écrits. Initialement, j'ai été fortement influencé par Richard A. Waterman, Rudi Blesh, Frederick Ramsey, Arthur M. Jones, Hugh Tracey, pour ce qui est du domaine des musiques africaines et afro-américaines; en histoire littéraire et en philosophie, ainsi qu'en psychologie, mes sources d'inspiration étaient Freud, Kafka, Schopenhauer, Jung, Stefan Zweig, George Orwell, David Riesman, Hans Prinzhorn (avec son ouvrage sur la peinture des «malades mentaux»), ou encore Ernest Kretschmer et la littérature populaire de science-fiction. 
A présent, il me semble significatif qu'après 1959 , je n'avais pratiquement plus d'attaches personnelles dans mon pays d'origine, hormis les relations de politesse. Le centre de mon engagement affectif s'était déplacé géographiquement aussi bien que culturellement. Dans les années 1959-1964, des personnalités africaines comme Evaristo Muyinda (Ouganda), Duro Ladipo (Nigeria), Hugh Tracey (Afrique du Sud) et A.M. Jones (également Africain à mes yeux) étaient devenues mes modèles; une série d'hommes plus jeunes, comme l'aveugle Waiswa Lubogo (Ouganda), Basilius Saprapason (Tanganyika) et Gboyega Ladipo (Nigeria) étaient devenus des amis admirés dans le sens d'une amitié entre pairs.

En 1964, vous avez fait la connaissance d'un couple de célèbres ethnologues et musicologues portugais, António et Margot Dias, qui allaient non seulement vous soutenir professionnellement mais aussi devenir vos amis. Vous êtes toujours en contact avec Margot, âgée maintenant de 85 ans.

C'est une lettre, reçue en 1964 du couple d'anthropologues António Jorge et Margot Dias à propos de mes recherches au Mozambique en 1962, qui marqua le début de ce contact fécond qui allait me permettre un accès durable au monde lusophone. En 1965, grâce à eux, j'ai obtenu une bourse de l'ancienne Junta de Investigações do Ultramar (actuellement Instituto de Investicação Cientifica Tropical) me permettant de faire du terrain pendant six mois en Angola. Rétrospectivement, je vois que cette recherche dans des contrées très reculées, tel l'ancien Distrito Cuando Cubango, ne représentait pas seulement une ethnologie d'urgence - car ces régions sont aujourd'hui plus ou moins inaccessibles en raison de la guerre civile - mais qu'elle s'est répercuté encore d'une manière décisive sur mon développement intellectuel. Sans m'en apercevoir, c'est en Angola que je me suis métamorphosé de musicologue en anthropologue culturel, si l'on veut l'appeler ainsi. C'est peut-être le fruit du hasard, car je n'étais parti avec aucun programme de recherche préétabli, ni orienté vers aucune problématique particulière. Par hasard, je me trouvais là au beau milieu d'une culture qu'il était impossible de comprendre sans $\mathrm{y}$ participer - participation qu'il faut entendre ici comme un véritable processus d'enculturation. Dans le sud-est de l'Angola, parmi des populations parlant des langues comme le mbwela, le nkhangala, le luchazi, le mbunda et le tchokwé, j’ai fait pour la première fois l'apprentissage systématique d'une langue africaine. Depuis 1959, je maîtrisais un peu le kiswahili, et j'avais acquis des notions de luganda et de yoruba (Nigéria), mais je ne parlais aucune langue africaine couramment. L'apprentissage du mbwela me permit un accès direct à cette aire culturelle; en même temps, j'ai découvert que je ne pouvais comprendre la culture sans me soumettre aux rites d'initiation. Dans l'ensemble, ces expériences pendant six mois de séjour en Angola ont modifié mon profil culturel personnel. Désormais, il existait une culture où je ne passais plus guère comme "étranger ». Ma proximité par rapport aux cultures de l'Angola oriental et du nord-ouest de la Zambie n'est pas seulement à l'origine de l'énorme intérêt que j'ai réservé par la suite à l'anthropologie cognitive; elle constitue aussi jusqu'à présent l'un des deux fondements de mon lien spirituel avec l'Afrique.

Ma recherche en Angola, de type participante et centrée sur l'enculturation et le processus de socialisation des enfants et adolescents des deux sexes, s'est élargie rapidement à une étude des représentations religieuses, des pratiques médicales locales et des problèmes psychologiques. En 1971, elle a donné lieu à une thèse de doctorat portant sur ce même thème. 
D'autre part, cette même recherche angolaise m'a amené à réorienter mes études à l'Université de Vienne et à opter dès 1968 pour l'ethnologie comme matière principale et la musicologie comparée comme matière à option. Ma thèse était d'orientation ethnologique.

Vous venez d'évoquer les deux fondements de votre lien spirituel avec l'Afrique. Le premier est votre recherche en Angola. Quel est le second?

Le second fondement de mon lien spirituel avec l'Afrique réside en Afrique australe orientale: il s'agit des frères Daniel et Donald Kachamba. Bien qu'une première rencontre avec ces deux musiciens et compositeurs de kwela ait eu lieu en 1967 déjà dans leur propre pays, au Malawi, nous n'avons repris contact qu'à partir de janvier 1972 lorsque, après six mois de terrain en Zambie, je leur ai rendu visite à nouveau. J'ai tant écrit sur le dérivé africain du jazz créé par les frères Kachamba qu'il n'est pas nécessaire d'entrer dans les détails ici [Kubik 1974] - si ce n'est pour rappeler que j'ai bientôt été intégré à leur formation musicale. J'ai aussi participé au cours de vingt années consécutives à des tournées de l'ensemble en diverses formations, me conduisant dans trente-trois pays d'Europe, d'Afrique et d'Amérique latine. Comme vous pouvez le deviner, l'appartenance à l'ensemble des frères Kachamba signifiait pour moi la reconquête complète de mon appartenance au monde du jazz quitté en 1959, mais sous une nouvelle forme. Depuis, je suis parfaitement heureux musicalement.

Nos nombreuses tournées et nos liens étroits ont fait que je me suis également intégré à la famille Kachamba, y compris la parenté proche, surtout la famille de Moya Aliya Malamusi. En 1967, âgé alors de huit ans, ce dernier avait été le joueur de hochet du groupe, avant d'apprendre la contrebasse monocorde. Adulte, il est devenu chercheur renommé dans le domaine de la littérature orale d'Afrique australe. Il n'est dès lors guère surprenant que grâce à ce second lien spirituel avec l'Afrique s'amorça une relation personnelle décisive : mon mariage avec Lidiya Malamusi, une sœur de Moya, relation d'abord informelle puis, peu avant sa mort en 1989, conclue formellement par un mariage. A partir de 1982, notre formation musicale se transforma partiellement en une équipe de chercheurs. Ce fut alors la période la plus féconde de ma vie du point de vue de la recherche, comme en témoigne la liste de mes publications à partir du début des années quatre-vingts. Jamais je n'ai autant travaillé et publié que de 1982 à 1989, soutenu par Lidiya.

Dans votre village de Singano, près de Chileka au Malawi, Moya dirige à présent un centre de littérature orale. En même temps, il a ouvert un petit musée ethnographique. Comment participez-vous à ces entreprises?

Je n'y participe pas directement. Les projets de recherche de Moya sur la littérature orale et la musique au Malawi et dans les pays limitrophes sont soutenus par divers organismes. Mais nous faisons du terrain ensemble, comme dans le cas de notre actuelle enquête, qui dure déjà depuis trois ans, sur la musique et la littérature orale en Namibie. Un aspect nouveau de notre comportement de terrain est que Moya amène régulièrement avec lui son fils, maintenant âgé de sept ans, dont la présence a un effet bénéfique sur nos contacts avec la population locale.

Voyons maintenant un autre aspect important de vos recherches dans le domaine de la culture. Comment s'est produit, en 1974, le passage de l'Afrique à l'Amérique du sud?

Ce fut à la suite de mon retour à la pratique musicale comme membre du groupe de jazz kwela des frères Kachamba. Dans le cadre de nos nombreuses tournées organisées par le 
Goethe-Institut, Donald Kachamba et moi-même nous sommes rendus au Venezuela et au Brésil, en 1974. En me souvenant de ce voyage d'une durée de plusieurs semaines, je m'aperçois à quel point l'année 1974 représenta une nouvelle étape décisive dans mon évolution personnelle. Imaginez-vous deux individualistes s'étant rapprochés à travers la musique et une amitié de longue date, mais ayant des perspectives divergentes en raison d'un tempérament différent. Alors imaginez-vous ces deux gars, l'un né en Afrique, l'autre en Europe, parachutés pour la première fois au Nouveau Monde. Cela donne un regard stéréo à quatre yeux. Notre réaction était intéressante. Le Venezuela nous a laissés plutôt froids; nous le trouvions trop rude, trop "espagnol», trop ibérique. Ce fut tout à fait différent au Brésil, où que nous allions, en particulier à Salvador de Bahia. Pour nous deux, c'était comme un retour au continent africain. Donald abandonna toute réserve. Bien des scènes turbulentes ont jalonné nos enregistrements de candomblé et de samba des rues dans la banlieue de Salvador. En 1974, dans un terreiro à Salvador, un adolescent a fait cadeau à Donald d'une bague symbolique en fer comme signe du lien spirituel avec l'Afrique; Donald l'a portée pendant tout le voyage.

L'année suivante (1975) nous vit à nouveau au Brésil pour une tournée. En 1980, lors de mon quatrième voyage de recherches et de conférences au Brésil, il y avait dans notre équipe Donald Kachamba, Moya A. Malamusi et le folkloriste brésilien Guilherme dos Santos Barbosa. Mes enquêtes de terrain au Brésil, menées soit en collaboration avec mes amis africains, soit en solitaire, ont surtout porté sur les banlieues de Salvador de Bahia, de Rio de Janeiro et de São Paulo, sur de petites villes comme Capivari (État de São Paulo) [Kubik 1990] et sur les zones rurales où j'ai essayé de faire des recherches sur les vestiges de langues africaines (Minas Gerais, État de São Paulo et Mato Grosso), qui ont donné lieu à diverses publications [e.g. Kubik 1979].

Par rapport aux conditions brésiliennes, la méthode de terrain adoptée par votre équipe était unique. Que pouvez-vous dire à ce sujet ? Quels étaient les objectifs de vos recherches au Brésil ?

Étant donné la composition de notre équipe, il était naturel que la recherche se concentrât sur ce que le panorama culturel brésilien comportait d'éléments africains reconnaissables. A ce sujet, le regard en stéréo, déjà évoqué, nous a amenés à un large consensus, à savoir que: 1 . sur la base de nos expériences dans de nombreux pays africains, nous supposions d'emblée, contrairement à un certain nombre de Brésiliens, une pluralité de cultures africaines, soulignant ainsi la réalité du pluralisme culturel en Afrique subsaharienne; 2. nous ne considérions pas la scène brésilienne comme un amas de survivances culturelles africaines en voie de disparition; plutôt, nous constations tout autour de nous des ramifications des cultures africaines qui nous étaient familières (dont celles de vastes régions angolaises et celles appartenant à l'aire de langue yoruba et fõ sur le littoral ouest-africain), jusqu'à la transplantation de cultures demeurées intactes et ayant fait preuve de leur vitalité.

Un autre constat s'est imposé à nous pendant nos recherches au Brésil : nous avons davantage pris conscience de l'historicité concomitante et tout à fait équivalente des cultures africaines par rapport à celles du Nouveau Monde, ce qui nous mettait en porte-à-faux par rapport au stéréotype fort répandu de la société prétendument traditionnelle. Comme toutes les autres cultures, celles de l'Afrique n'ont cessé de se transformer au cours de leur histoire - processus qui n'est pas seulement typique du XIX ${ }^{e}$ siècle. La tradition, l'emprunt et l'innovation engendrent à chaque fois de 
nouvelles configurations. Nous en avons conclu, entre autres, que l'étude des cultures afro-brésiliennes et de leurs rapports historiques avec les cultures africaines exigent le recours aux mêmes méthodes historiques de part et d'autre de l'Atlantique.

Venons-en aux institutions scientifiques en Europe. Comment voyez-vous aujourd'hui la formation universitaire en ethnomusicologie?

Voilà une question à laquelle j'ai de la peine à répondre. Ma préoccupation centrale a toujours été la recherche. Je passe six ou sept mois par an en Afrique. Je ne suis donc impliqué que marginalement dans l'enseignement supérieur en Europe, tout au plus quatre heures hebdomadaires pendant un seul semestre par an. Mais il faut préciser qu'en Afrique même, j'ai pu transmettre à plusieurs personnes une formation méthodologique et technique de l'anthropologie culturelle, dans le cadre de recherches pratiques sur le terrain. Dans ce sens, Moya Aliya Malamusi, Maurice Djenda, Mose Yotamu, Danhin Amagbenyõ Kofi, Lidiya Malamusi ( $†$ ), Marcelina Gomes et d'autres ont été mes « étudiants », mes « étudiants privés » si vous voulez.

En Europe, les systèmes d'éducation ont une orientation profondément collectiviste, dans le sens de l'université de masse - constat qui s'applique également, bien sûr, aux universités africaines créées sur le modèle européen. Mais dans les zones rurales en Afrique, par ma propre initiation à diverses institutions (par exemple: mukanda, mungonge et vandumbu en Angola, nyau au Malawi), j'ai fait l'expérience de systèmes de formation organisés de façon nettement plus individualiste. En Angola et au nord-ouest de la Zambie, dans l'institution de circoncision appelée mukanda, le personnel enseignant est beaucoup plus nombreux que les tundanda (garçons à initier). Chaque kandanda est doublement parrainé, par le chilombola et son assistant (kalombola-tito). De plus, dans l'école mukanda, le fondateur ou directeur (chizika-mukanda) est lui-même actif, ainsi que ceux qui enseignent la musique, la danse et la récitation (ngomba), assistés à leur tour par une multitude d'hommes adultes du village. Dans les cultures occidentales - qui ne manquent pas de qualifier les cultures africaines de « collectivistes »- rare est l'individu auquel on porte tant d'attention...

Quelles sont les principales méthodes de terrain que vous avez élaborées et appliquées ?

Vue en rétrospective, ma principale méthode de terrain a été ce que j'ai appelé le random drifting («se laisser aller au hasard»). Cela ne m'empêche pas pour autant d'emporter toujours avec moi quantité de questions et de problèmes à résoudre. Le terme de random drifting se réfère à la méthode de "naviguer" sur le terrain. En 1959/60, quand j'ai voyagé en autostop et à pied dans onze pays africains, ou encore en 1964, quand j'ai traversé à vélosolex tout le nord de l'Afrique centrale à partir du Nigeria, j'ai fait mon expérience initiale de cette méthode, sans l'avoir désignée de la sorte. C'est exactement l'opposé de ce que les projets de terrain conçus dans le cadre des institutions universitaires européennes exigent des étudiants et des chercheurs débutants. On leur demande une approche orientée vers une problématique. Malheureusement, une telle problématique est bien souvent préfabriquée. Ainsi prendon pour point de départ des problèmes fantômes. Sous l'effet de mes premières expériences africaines, j'ai vite compris qu'on ne construit pas une maison en commençant par le toit. Ma méthode consiste à découvrir les problèmes sur le terrain même, pendant que je me laisse porter par le hasard. Il va sans dire que ces problèmes ne peuvent pas tous être résolus dans l'immédiat, qu'il faut en laisser certains pour plus tard. 
Ce que j'ai souvent perçu comme un obstacle à la recherche scientifique, c'est l'exigence de réaliser un programme préétabli comme le symbolise notamment le questionnaire rédigé à l'avance, rempli de questions qui s'avèrent souvent inapplicables sur place. D'autre part, un programme préconçu, voire une approche orientée vers une problématique spécifique constitue un obstacle dans la mesure où la situation sur le terrain peut changer rapidement, à l'image du trou dans la couche d'ozone sur une partie de notre planète. Toute fixation est susceptible de contrarier. Celui qui se fixe l'objectif de se rendre en Angola en 1993 pour y étudier l'histoire des cloches doubles passera sans doute à côté de découvertes inouïes ; il suffit de lire mon ami Moya Aliya Malamusi [1991 : 55-56] pour savoir comment le chercheur de terrain doit se comporter s'il ne trouve pas exactement ce qu'il cherche...

J'ai moi-même quitté l'Europe en 1959 à la recherche, pour ainsi dire, des « racines » du jazz. En Ouganda, j'ai trouvé autre chose. En 1965, je me suis rendu en Angola pour étudier la musique du sud-est du pays. J’ai fini par étudier l'initiation. Et ainsi de suite.

Néanmoins, j'applique des méthodes de terrain plus spécifiques, se rapportant à des activités aussi diverses que l'enregistrement sonore, la communication sociale, le tournage d'un film, la transcription, l'analyse comparée d'ordre organologique et technologique et l'apprentissage d'une langue... Mon approche théorique ne recourt toutefois pas à la création de concepts en langue étrangère, censés traduire les données collectées sur place. Il n'est donc guère surprenant que j'ai rayé de mon vocabulaire scientifique de nombreuses notions ayant cours en ethnologie - ce que je recommanderais d'ailleurs à tout un chacun: notre science - y compris l'ethnomusicologie - avec son vocabulaire démesuré et confus en langues européennes a définitivement besoin de mesures anti-inflationnistes.

Il est vrai que vous avez rayé des notions courantes de votre vocabulaire, mais en même temps vous en avez créé d'autres, dont certaines sont entrées dans la terminologie spécialisée. Toutefois, cette terminologie restant liée aux objets de vos recherches, elle ne devient pas une fin en soi. Que pensez-vous en principe de la construction théorique?

Peut-être qu'une différence fondamentale entre mon approche et celle de quelques-uns de nos collègues réside dans le fait que ces derniers tendent à bâtir des théories et des concepts au préalable, pour procéder de manière déductive, en recueillant des données venant confirmer ou infirmer leurs notions théoriques. En revanche, mon approche consiste à découvrir des théories.

D'une manière générale, mes théories s'élaborent de bas en haut, elles procèdent de l'étude et de l'analyse des données. Ce sont les données empiriques qui me guident vers la théorie. En voici un exemple qui doit vous être familier, puisque c'est vous qui en avez fait la démonstration lors de la $32^{\mathrm{e}}$ conférence mondiale de l'International Council for Traditional Music (ICTM) à Berlin, en juin 1993. Dans une large mesure, votre démonstration correspond à une approche théorique qui est aussi importante pour moi. Voici votre exemple : lors d'enquêtes sur le terrain au Brésil, vous avez fait écouter à un ensemble de musiciens de capoeira dans la région du Recôncavo (Bahia), l'enregistrement d'un joueur d'arc musical angolais, José Virasanda, qui appartient à une tradition dont on sait qu'elle possède des ramifications au Brésil. Votre intention originelle était de découvrir, sur la base des réactions de vos auditeurs, si ceux-ci percevaient des similitudes et des continuités historiques. Ce faisant, vous partiez bien évidemment d'un théorème, mais ce n'est pas important pour la démonstration. Ce qui 
est important, c'est que le comportement des gens auxquels vous faisiez écouter l'enregistrement angolais n'apporta aucune réponse valable au problème que vous aviez conçu, car vos auditeurs ne l'avaient pas conçu à leur tour. Par contre, leur comportement vous a fait découvrir des aspects théoriques inattendus: 1. que la confrontation avec l'exemple angolais ne sollicitait aucune réponse chez les musiciens de capoeira susceptible de vous révéler la conception intra-culturelle et la structure de la musique de l'Angolais Virasanda. C'est là un résultat crucial, mettant en relief le caractère fermé des systèmes cognitifs : vos auditeurs étaient incapables de deviner le système de Virasanda. 2. Vous avez découvert avec autant d'étonnement que le comportement de votre groupe brésilien faisait ressortir des aspects essentiels et profonds de leur propre univers de représentations, abordé sous l'angle émique. 3. Il est apparu enfin que les gens vous fournissaient indirectement un fil conducteur que vous pouviez suivre lors de recherches futures en Angola.

Si vous vous mettiez à tester de manière analogue le même document sonore ou des documents différents avec d'autres groupes au Brésil, les résultats obtenus équivaudraient à un échantillon représentatif. Si de telles enquêtes donnaient un résultat semblable, l'étape suivante consisterait à formuler une hypothèse de travail du genre : si on fait X, on obtient Y1, Y2, Y3 comme réaction. Et ainsi de suite. Lentement, vous seriez ainsi arrivé à un modèle théorique. C'est à cela que je me réfère en disant que des théories se laissent découvrir : tout se passe comme si ces théories étaient déjà préfigurées dans l'ordre expérimental des données, semblables aux empreintes d'un fossile ; ainsi, on les dé-couvre. Somme toute, il s'agit de dégager le modèle théorique du tout avec précaution. Ce n'est donc pas une construction.

Y a-t-il d'autres procédés pour dé-couvrir des théories existant dans la culture mais invisibles pour le chercheur?

Oui, il existe une autre manière de découvrir des théories. Mais je ne pense ici ni aux théories indigènes dans le sens de l'ethnoscience des années soixante, ni à la nouvelle ethnographie entendue comme la découverte de systèmes cognitifs identifiables sous l'angle émique. C'est en nous-mêmes que nous pouvons découvrir des théories, où que nous nous trouvions. Car nous avons en nous des instances qui traitent les données de manière autonome jusqu'à en arriver à des constellations, à des agencements et à des interrelations dont nous ne prenons pas conscience au demeurant. Puis, subitement, le résultat surgit, et nous voilà devant une idée tout à fait surprenante. Pour ce qui me concerne, la découverte de théories en moi-même résulte d'un processus qui ressemble à une forme d'écoute interne, comme on écouterait une pièce pour xylophone amadinda en prêtant une attention particulière à sa dimension profonde. Pareille écoute suscite des schémas inhérents, dont la perception est automatique. Autrement dit, dans bien des cas, je cède à mon inconscient la tâche de mise en ordre qu'exige la construction théorique, pour découvrir ensuite avec étonnement ce qui voit le jour.

Dès le début de vos voyages en Afrique, vous avez systématiquement réalisé des enregistrements sonores, puis visuels de la pratique musicale, des fêtes, mais aussi de la vie quotidienne dans les cultures africaines. En quoi consistent aujourd'hui vos collections ? Qu'en reste-t-il à évaluer ? Combien de temps vous faudrait-il pour y parvenir?

Étant donné que ces trente dernières années, j'ai passé deux tiers environ de mon temps en Afrique pour la recherche, une collection assez importante de matériaux s'est accumulée dans mes archives. Celles-ci comprennent environ 36000 enregistrements sur bande magnétique, environ 20000 photographies, de nombreuses heures de film 
16mm, plusieurs jours de matériel filmé 8mm depuis 1962 et, à partir de 1990, une collection de films vidéo S-VHS (22 cassettes de 45 minutes chacune jusqu'à présent). S'y ajoutent de nombreux cahiers de terrain reliés et dactylographiés, trois à quatre cents pages pour chaque séjour, et les journaux de terrain sténographiés fort précieux dont le premier remonte à un voyage en Finlande en 1958.

... ce qui fait de votre collection les archives privées les plus importantes de musique et de littérature orale africaines. Comment concevez-vous votre responsabilité dans la " gestion », la conservation et l'analyse d'archives si volumineuses?

J'ai pris conscience du danger après une explosion de gaz inattendue, provoquée en octobre passé par un suicidaire dans l'immeuble en face de mes archives, ayant entraîné d'importants dégâts dans mon appartement (mais heureusement sans entamer mes documents scientifiques) et causé la mort d'une femme âgée. Je n'ai pas l'habitude de faire de beaux discours sur l'éthique de la science. Mais, admettons-le, j'ai une grande responsabilité que je me suis imposée moi-même : jamais un chercheur ne doit détruire ou laisser détruire ce qu'il a collecté. Cela s'applique aux enregistrements sonores et aux documents visuels autant qu'aux notes personnelles. Les exigences de conservation et d'analyse de mon propre matériel scientifique ont acquis une dimension telle que je ne puis les assumer entièrement, ni financièrement ni dans le temps qui me reste. Pour analyser et fixer par écrit seulement les résultats de mes enquêtes, il me faudrait quarante ans au moins. Cela explique pourquoi je refuse depuis des années toutes les chaires universitaires qui me sont proposées. Je considère que ma tâche principale consistera à faire avancer la recherche. Voici la liste des projets de publication les plus urgents :

- a)Nthano. Récits en chichewa/chinyanja, Malawi (transcription intégrale des contes par ma femme Lidiya Malamusi, provenant de ses fonds augmentés par des récits enregistrés sur le terrain depuis 1989).

- b)La littérature orale dans les langues de l'Angola oriental (ouvrage en plusieurs volumes, en édition bilingue, comprenant des transcriptions et des interprétations de tous les textes relevés dans cette aire culturelle depuis 1965 ; à rédiger en collaboration avec Mose Yotamu, Fortunato Pereira Gonçalves et d'autres).

- c)L'initiation mungongi en Angola (témoignages).

- d)L'expérience de mukanda. Étude comparative de la psychologie initiatique dans trente-sept écoles de circoncision mukanda (Angola, Zambie).

- e)Angome iboga. Témoignage d'un prêtre du bwiti au Gabon (sur la base de recherches menées en 1964, 1966 et 1970).

Pour conclure, que pourriez-vous dire à propos de votre journée de travail, et quels conseils aimeriez-vous donner aux jeunes chercheurs?

Un aspect important de ma technique de travail consiste à me laisser aller à mon inspiration, sans tenir compte des délais. C'est seulement ainsi que l'on accomplit le maximum de la meilleure façon. Si je dois m'acharner à rédiger un article dont le sujet m'a été imposé (et plus les sujets sont d'ordre général, plus ils me déplaisent), il se peut que je n'obtienne rien pour finir. Alors il vaut mieux laisser tomber tout cela et terminer ce vers quoi on est porté. C'est donc ma propre force motrice qui me fait progresser. Les plus grands inconvénients qui m'empêchent de m'acquitter d'une tâche scientifique dans les meilleures conditions sont les interruptions: chacune provoque une perte de temps, il faut beaucoup d'énergie pour se replonger mentalement dans le sujet auquel on a été arraché. Ce sont donc ces prises de conscience qui déterminent ma 
méthode de travail. Un autre secret de la productivité scientifique réside dans la limitation de la sociabilité au strict minimum.

Ma dernière question concerne la motivation qui vous a fait travailler tout au long de votre vie comme rarement un anthropologue culturel l'a fait. Elle se résume à un seul mot: pourquoi?

Ce « pourquoi » ne peut être saisi. En dernière instance, il est transcendant.

\section{BIBLIOGRAPHIE}

FÖDERMAYR Franz, Dietrich SCHÜLLER \& August SCHMIDHOFER éd., 1994, Festschrift für Gerhard Kubik. Frankfurt/Bern : Peter Lang.

KUBIK Gerhard, 1960, « Als Autostopper im unabhängigen Congo ». Neue Zürcher Zeitung, Nr. 209, Blatt 4, Sonntag 31. Juli 1960.

KUBIK Gerhard, 1974, The Kachamba Brothers Band. A Study of Neo-Traditional Music in Malawi. Lusaka : Institute for African Studies, UNZA.

KUBIK Gerhard, 1979, Angolan Traits in Black Music, Games and Dances of Brazil. A Study of African Cultural Extensions Overseas. Estudos de Antropologia Cultural $N^{\circ} 10$. Lisboa : Junta de Investigações Científicas do Ultramar.

KUBIK Gerhard, 1990, « Drum patterns in the batuque of Benedito Caxias ». Latin American Music Review 11(2) : 115-181.

KUBIK Gerhard, 1991, Extensionen afrikanischer Kulturen in Brasilien. Aachen : Alano.

KUBIK Gerhard, 1992, « Embaire xylophone music of Samusiri Babalanda (Uganda 1968). The World of Music 34(1) : 57-84.

KUBIK Gerhard, 1994, Theory of African Music, vol. 1. Berlin : Institute for Traditional Music (Intercultural Music Studies 7).

KUBIK Gerhard \& Helmut ANDIKA, 1967, Gedichte und Prosa - Zeichnungen und Aquarelle. Wien : Verlag Junge Generation.

MALAMUSI Moya Aliya, 1991, « Samba ng'oma eight. The drum chime of Mario Sabuneti ». African Music 7(1) : 55-71.

\section{NOTES}

*. Traduit de l'allemand par Isabelle Schulte-Tenckhoff.

1. Voir aussi ma réaction émotionnelle aux événements survenant au lendemain de l'indépendance du Congo Belge que j'ai traversé en 1960; cf. Kubik (1960) et le courrier des lecteurs paru dans les numéros suivants de la Neue Zürcher Zeitung.

2. Cf. « Letters to the Editor », African Music, vol. 2, no. 4, 1961. 